\title{
RELIABILITY ANALYSIS OF FOOTSCAN PLATE SYSTEM IN MEASUREMENT OF PLANTAR PRESSURE OF SPASTIC DIPLEGIA CHILDREN
}

\section{ANALIZA FIABILITĂTII SISTEMULUI DE SCANARE A PICIORULUI LA MĂSURAREA PRESIUNII PLANTARE LA COPIII CU DIPLEGIE SPASTICĂ}

\author{
Ting $\mathrm{LI}^{1}$, Binqian $\mathrm{LI}^{1}$, Wuyong $\mathrm{CHEN}^{1}$, Jianxin $\mathrm{WU}^{2}$, Bo XU${ }^{1}$, Jin $\mathrm{ZHOU}^{1,2^{*}}$ \\ ${ }^{1}$ National Engineering Laboratory for Clean Technology of Leather Manufacture, Sichuan University; Chengdu, P. R. China \\ ${ }^{2}$ Science Lab, Zhejiang Red Dragonfly Footwear Co., LTD., Zhejiang Province, Wenzhou 325100, P. R. China
}

RELIABILITY ANALYSIS OF FOOTSCAN PLATE SYSTEM IN MEASUREMENT OF PLANTAR PRESSURE OF SPASTIC DIPLEGIA CHILDREN

ABSTRACT. This study was aimed at evaluating the reliability of Footscan plate system when measuring pressure in spastic diplegia children (CP). Eighteen CP and 36 typical developed counterparts (TD) with similar age, height and weight were recruited and their plantar pressure was measured in at least five trials. Three-mask model was applied and plantar regions were divided into: forefoot, mid foot and hind foot, under which peak pressure (PP) (N/ $\left.\mathrm{cm}^{2}\right)$, relative pressure time integral (PTIrel) (\%) and relative contact area (CArel) (\%) were calculated. Coefficient of variance (CoV) and intra class coefficient (ICC) were explored for each variable and region. Our outcomes show that in terms of CP, with the increasing of test times, ICC of PP increased, while those of PTIrel and CArel were lowered; additionally, ICC of TD of each pressure variable successively improved. A similar tendency was found for CoV. Considering entire feet of CP and five measurements, reliability of CArel was the best in the three areas; whereas PP and PTIrel were demonstrated to be less reliable, except for the midfoot area. In contrast, reliabilities of TD were shown to be good for all pressure variables under all three regions. Overall, reliability of Footscan plate system in the study of CP was moderate in most variables and under most plantar regions. Thereby, by increasing the test times to no less than five, this protocol could be applied in the clinical and scientific investigation of CP children.

KEY WORDS: spastic diplegia children, plantar pressure, reliability, intra class coefficient, coefficient of variance

\section{ANALIZA FIABILITĂTII SISTEMULUI DE SCANARE A PICIORULUI LA MĂSURAREA PRESIUNII PLANTARE LA COPIII CU DIPLEGIE SPASTICĂ}

REZUMAT. Acest studiu a avut ca scop evaluarea fiabilităţii sistemului Footscan la măsurarea presiunii la copii cu diplegie spastică (PC). S-au recrutat 18 subiecţi cu PC şi 36 de subiecţi cu dezvoltare normală (TD), cu vârstă, înălţime şi greutate similare şi s-a măsurat presiunea plantară de cel puţin cinci ori. S-a aplicat modelul cu trei zone de analiză şi regiunile plantare au fost împărţite astfel: antepicior, partea mediană şi retropicior, unde s-au calculat presiunea maximă (PP) (N/cm²), integrala relativă presiune-timp (PTIrel) (\%) şi zona de contact relativă (CArel) (\%). S-au explorat coeficientul de variaţie (CoV) şi coeficientul intra-clasă (ICC) pentru fiecare variabilă şi regiune. Rezultatele noastre arată că, în ceea ce priveşte subiecţii cu PC, la mărirea numărului de măsurători, ICC al PP a crescut, în timp ce ICC al PTIrel şi CArel a scăzut; în plus, ICC s-a îmbunătătit succesiv la subiecţii din grupa TD la fiecare variabilă de presiune. O tendinţă similară s-a constatat pentru CoV. La analiza piciorului întreg la subiecţii cu PC şi la efectuarea măsurătorilor de cinci ori, fiabilitatea CArel a fost cea mai bună în cele trei regiuni, iar PP şi PTIrel s-au dovedit a fi mai puţin fiabile, cu excepţia regiunii mediane a piciorului. În schimb, valorile fiabilităţii la grupa TD s-au dovedit a fi bune pentru toate variabilele de presiune în toate cele trei regiuni. În general, fiabilitatea sistemului Footscan la studierea subiecţilor cu PC a fost moderată pentru majoritatea variabilelor şi în majoritatea regiunilor plantare. Astfel, prin creşterea numărului de măsurători la nu mai puţin de cinci, acest protocol ar putea fi aplicat în investigaţia clinică şi ştiinţifică a copiilor cu PC.

CUVINTE CHEIE: copii cu diplegie spastică, presiune plantară, fiabilitate, coeficientul intra-clasă, coeficientul de variaţie

L'ANALYSE DE LA FIABILITÉ DU SYSTÈME FOOTSCAN EN MESURANT LA PRESSION PLANTAIRE CHEZ LES ENFANTS DIPLÉGIQUES SPASTIQUES

ABSTRAIT. Cette étude a eu le but d'évaluer la fiabilité du système Footscan en mesurant la pression chez les enfants diplégiques spastiques (CP). On a recruté dixhuit sujets avec CP et 36 sujets développés normalement (TD) avec l'âge, le poids et la taille similaires et on a mesuré leur pression plantaire d'au moins cinq essais. On a appliqué le modèle à trois régions d'analyse et les régions plantaires ont été divisées comme suit: avant-pied, médio-pied et arrière-pied, où on a calculé la pression maximale (PP) (N/ $\mathrm{cm}^{2}$ ), l'intégrale relative pression-temps (PTIrel) (\%) et la zone de contact relative (CArel) (\%). On a exploré le coefficient de variance (CoV) et le coefficient intra-classe (ICC) pour chaque variable et région. Nos résultats montrent que, en ce qui concerne les sujets avec $\mathrm{CP}$, avec l'augmentation du nombre de mesures, I'ICC de PP est augmenté, tandis que I'ICC de PTIrel et CArel ont abaissé; en outre, I'ICC de chaque variable de pression chez le sujets TD est successivement amélioré. On a trouvé une tendance similaire pour CoV. En considérant le pied entier chez les sujets avec CP et en mesurant cinq fois, la fiabilité de CArel a été la meilleure dans les trois régions; tandis que le PP et PTIrel ont été moins fiables, sauf pour le médio-pied. D'autre part, la fiabilité chez le sujets TD a été bonne pour toutes les variables de pression dans les trois régions. Dans l'ensemble, la fiabilité du système Footscan dans l'étude de CP a été modérée dans la plupart des variables et dans la plupart des régions plantaires. Ainsi, en augmentant les mesures à pas moins de cinq, ce protocole pourrait être appliqué dans la recherche clinique et scientifique des enfants avec CP.

MOTSCLÉS: enfants diplégiques spastiques, pression plantaire, fiabilité, coefficient intra-classe, coefficient de variance

* Correspondence to: Jin ZHOU, National Engineering Laboratory for Clean Technology of Leather Manufacture, Sichuan University; Chengdu 610065, P. R. China; e-mail: zj_scu@qq.com 


\section{INTRODUCTION}

Feet are the only part of the human body coming into contact with the ground and they transfer the ground reaction force to the bones and muscles of the lower and upper body, in order to generate enough moment and momentum to propulse the body moving forward [1]. Nowadays, the pressure plate system has been widely used in the study of foot biomechanics for plantar pressure measurement, in which ground vertical reaction forces were recorded $[2,3]$. Since voluntary movement and irregular motion of children with cerebral palsy (CP) were commonly observed in their clinical inspection [4-7], those uncertainties might diminish the reliability and validity of outcomes of plantar pressure distribution of CP. Thereby effectiveness of Footscan plate system in measurement of plantar pressure of $\mathrm{CP}$ should be quantitatively evaluated.

Current literature shows that in terms of measuring healthy subjects, Novel and Tekscan plate system had been approved to be with a medium to good reliability and repeatability [1-3, 8]. Meanwhile, Low and Dixon [9] used Footscan insole system to measure and analyze the pressure distribution at fore and hind foot; then they confirmed that this insole system had a good repeatability. In addition, De Cock [10] also reported that Footscan plate system was a reliable protocol in the foot pressure acquisition. Moreover, Jin et al. [11-13] applied pressure plate for the study of the plantar pressure distribution of healthy children.

However, in terms of $\mathrm{CP}$, the focus was set on the study of reliability of temporal-spatial protocols $[6,14,15]$. Further, only Jacques [16] studied the reliability of pressure measurement of typical developed children with foot deformity. But the foot deformities were only one of the factors affecting the gait stability, other abnormalities in terms of central nervous and muscular control were more critical. As a kind of $C P$, spastic diplegia (CP) influenced both lower limbs in terms of muscle control and coordination whilst walking, hence it was important to explore the features of $\mathrm{CP}$ in the issues of balance and mechanism of loading such as

\section{INTRODUCERE}

Picioarele reprezintă singura parte a corpului uman care intră în contact cu solul şi transferă forţa de reacţie a solului la oasele şi muşchii corpului inferior şi superior, pentru a genera suficient moment şi impuls de propulsie a corpului în mişcare [1]. În zilele noastre se utilizează pe scară largă placa de presiune pentru studiul biomecanicii piciorului prin măsurarea presiunii plantare, care înregistrează forţele de reacţiune verticale ale solului [2, 3]. Întrucât în inspecţia clinică s-au observat frecvent mişcările involuntare şi neregulate ale copiilor cu paralizie cerebrală (PC) [4-7], aceste incertitudini ar putea diminua credibilitatea şi validitatea rezultatelor de distribuţie a presiunii plantare la copiii cu PC. Prin urmare, trebuie să se evalueze cantitativ eficienţa sistemului Footscan la măsurarea presiunii plantare la copiii cu PC.

Studiile actuale au arătat că, în ceea ce priveşte măsurarea subiecţilor sănătoşi, sistemele cu placă de forţă de tip Novel şi Tekscan au fost aprobate, considerate cu fiabilitate şi repetabilitate medii spre bune $[1-3,8]$. Low şi Dixon [9] au utilizat sistemul Footscan pentru a măsura şi analiza distribuţia presiunii la antepicior şi retropicior, confirmând că acest sistem a avut o repetabilitate bună. În plus, De Cock [10] a raportat, de asemenea, fiabilitatea protocolului sistemului Footscan la măsurarea presiunii piciorului. Mai mult, Jin şi colab. [11-13] au utilizat placa de presiune pentru studierea distribuţiei presiunii plantare la copii sănătoşi.

Cu toate acestea, în ceea ce priveşte PC, accentul $s$-a pus pe studiul fiabilităţii protocoalelor temporalspaţiale [6, 14, 15]. Mai mult, doar Jacques [16] a studiat fiabilitatea de măsurare a presiunii la copii dezvoltaţi normal cu deformări la nivelul piciorului. Însă deformările picioarelor au reprezentat doar unul dintre factorii care afectează stabilitatea mersului, alte anormalităţi privind sistemul nervos central şi controlul muşchilor fiind mai critice. Ca un tip de PC, diplegia spastică $(P C)$ influenţează ambele membre inferioare în ceea ce priveşte controlul muscular şi coordonarea în timpul mersului, prin urmare, a fost important să se exploreze caracteristicile PC în probleme de echilibru şi 
the pressure distribution and gait cycle. Unfortunately, few studies were concerned with reliability of plantar pressure measurement of $C P$, and the lack of such basic information might hamper the explicit comprehension of the mechanism of loading for those subjects.

Thereby, our study was aimed at systemically evaluating the reliability of Footscan plate system in the pressure measurement of children with CP. According to literature, three hypotheses were proposed for CP: 1) as the number of tests increased, reliability of plantar pressure measurement would be improved gradually; 2) reliability would vary with the plantar regions, where pressure distribution at mid and forefoot would be more reliable than that at hindfoot; 3 ) reliability would vary with the pressure variables, where contact area would be better than peak pressure and pressure time integral.

\section{MATERIALS AND METHODS}

\section{Subjects}

Eighteen spastic diplegia children (CP) and 36 typical developed counterparts (TD) with similar age, height and weight were recruited. The inclusion criteria were shown below: (1) Gross Motor Function Classification System (GMFCS) level less than 3; (2) walking independently without support including orthopedic instrument; (3) cooperating in the whole test and completing all the measurements; (4) at least 5 trials being successfully recorded. Subjects' demographic information is given in Table 1. GMFCS was evaluated by an experienced clinician. The aims and methods of this test were first explained to patients' parents and a formal approval was obtained before the test. Furthermore, this study was approved by the ethics committee of university and the whole procedure followed the principles of Helsinki Declaration. mecanism de încărcare, cum ar fi distribuţia presiunii şi ciclul de mers. Din păcate, s-au efectuat foarte puţine studii cu privire la fiabilitatea de măsurare a presiunii plantare la persoane cu PC, lipsa acestor informaţii de bază putând împiedica înţelegerea explicită a mecanismului de încărcare la aceşti subiecţi.

Astfel, studiul nostru a avut ca scop evaluarea sistemică a fiabilităţii sistemului Footscan la măsurarea presiunii piciorului la copiii cu PC. Conform literaturii, sau propus trei ipoteze pentru PC: 1) pe măsură ce se măreşte numărul de teste, fiabilitatea măsurării presiunii plantare se îmbunătăţeşte treptat; 2) fiabilitatea variază în funç̧ie de regiunile plantare, distribuţia presiunii la antepicior şi în partea mediană a piciorului fiind mai fiabilă decât la retropicior; 3) fiabilitatea variază în funcţie de variabilele de presiune, zona de contact fiind mai bună decât presiunea maximă şi integrala timp-presiune.

\section{MATERIALE ŞI METODE}

\section{Subiecţi}

S-au recrutat optsprezece copii cu diplegie spastică (PC) şi 36 de subiecţi dezvoltaţi normal (TD), cu vârstă, înălţime şi greutate similare. Criteriile de includere sunt prezentate mai jos: (1) gradul de clasificare a funcţiei motorii grosiere (GMFCS) mai mic decât 3; (2) capacitatea de a merge în mod independent, fără sprijin, inclusiv fără instrumente ortopedice; (3) cooperarea pe tot parcursul testului şi finalizarea tuturor măsurătorilor; (4) înregistrarea cu succes a cel puţin 5 teste. Datele demografice ale subiecţilor sunt prezentate în Tabelul 1. GMFCS a fost evaluat de către un clinician cu experienţă. Obiectivele şi metodele acestui test au fost explicate în primul rând părinţilor pacienţilor şi s-a obţinut o aprobare oficială din partea acestora înainte de test. Mai mult decât atât, acest studiu a fost aprobat de către comitetul de etică al Universităţii şi toate procedurile au respectat principiile declaraţiei de la Helsinki. 
Table 1: Demographic information of subjects

Tabelul 1: Date demografice ale subiecţilor

\begin{tabular}{|c|c|c|}
\hline & TD & $\begin{array}{l}C P \\
P C\end{array}$ \\
\hline $\mathrm{N}$ & 36 & 18 \\
\hline $\begin{array}{c}\text { Age } \\
\text { Vârstă }\end{array}$ & $6.4 \pm 2.4$ & $6.6 \pm 3.0$ \\
\hline $\begin{array}{l}\text { Gender } \\
\text { Sex }\end{array}$ & $\begin{array}{l}M=18 \\
F=18\end{array}$ & $\begin{array}{l}M=12 \\
F=6\end{array}$ \\
\hline $\begin{array}{l}\text { Height (m) } \\
\text { Inălţime (m) }\end{array}$ & $1.1 \pm 1.7$ & $1.1 \pm 1.6$ \\
\hline $\begin{array}{l}\text { Weight }(\mathrm{kg}) \\
\text { Greutate }(\mathrm{kg})\end{array}$ & $22.8 \pm 7.7$ & $21.2 \pm 7.7$ \\
\hline $\begin{array}{l}\mathrm{BMI} \\
\mathrm{IMC}\end{array}$ & $16.8 \pm 2.4$ & $17.0 \pm 3.5$ \\
\hline GMFCS & $\begin{array}{l}\text { None } \\
\text { Niciuna }\end{array}$ & $\begin{array}{c}\text { Level I = } \\
\text { NiveluI I = } 4 \\
\text { Level II = } 8 \\
\text { NiveluI II = } \\
\text { Level III = } 6 \\
\text { NiveluI III = } 6\end{array}$ \\
\hline
\end{tabular}

\section{Pressure Measurement}

Children's plantar pressure was measured by Footscan pressure plate (one meter plate, RSscan Int., Belgium). The scanning frequency of this system is 250 $\mathrm{Hz}$, pressure sensor density is $4 / \mathrm{cm}^{2}$, and the range of measure is $0-200 \mathrm{~N} / \mathrm{cm}^{2}$. A two-step initial protocol was performed by the children and they were guided walking with their selected speed through the pressure plate which was located in the middle of six-meter-long track [16]. The protocol of two-steps has been proved to effectively reduce the times of trial and at the same time to improve the repeatability of the gait [17]. Before the measurement, the system was calibrated; and then three to five minutes warm up was provided. At least five successful measurements for each side of foot were also required.

\section{Măsurarea presiunii}

Presiunea plantară a copiilor a fost măsurată utilizând placa de presiune Footscan (placă de un metru, RSscan Int., Belgia). Frecvenţa de scanare a acestui sistem este de $250 \mathrm{~Hz}$, densitatea senzorului de presiune este de $4 / \mathrm{cm}^{2}$, iar gama de măsurare este 0 $200 \mathrm{~N} / \mathrm{cm}^{2}$. Copiii au urmat un protocol iniţial în două etape şi au fost îndrumaţi să meargă cu o viteză la alegere pe placa de presiune, care a fost poziţionată în mijlocul pistei de şase metri lungime [16]. Protocolul în două etape a redus efectiv durata procesului şi, în acelaşi timp, a îmbunătăţit repetabilitatea mersului [17]. Înainte de măsurare, sistemul a fost calibrat, apoi s-au alocat trei până la cinci minute de încălzire. De asemenea, a fost nevoie de cel puţin cinci măsurători reuşite pentru fiecare zonă a piciorului.

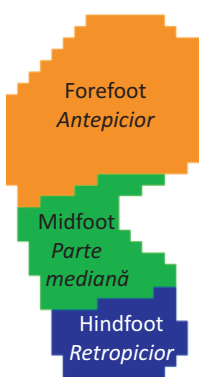

Figure 1. Three-mask model for plantar pressure analysis

Figura 1. Modelul cu trei zone pentru analiza presiunii plantare 


\section{Data Processing}

Three-mask model was applied and plantar regions were redefined by software of the system: forefoot (including five toes and five metatarsals), mid foot and hind foot. Then parameters of peak pressure (PP) $\left(\mathrm{N} / \mathrm{cm}^{2}\right)$, pressure time integral (PTI) $\left(\mathrm{S}^{*} \mathrm{~N} / \mathrm{cm}^{2}\right)$ and contact area (CA) $\left(\mathrm{cm}^{2}\right)$ were calculated under each area. In order to compare within the group, a normalization process was carried out for $C A$ and PTI: Relative CA $($ CArel $)=100 \times(C A(X i) / \Sigma C A(X i))$; relative $\mathrm{PTI}(\mathrm{PTI}$ rel $)=100 \times(\mathrm{PTI}(\mathrm{Xi}) / \Sigma \mathrm{PTI}(\mathrm{Xi}))$, where $\mathrm{Xi}$ indicates one of plantar regions.

\section{Statistical Analysis}

First of all, Q-Q Plot showed that all data tested followed the normal distribution; meanwhile results of independent $T$ test confirmed that no significant differences existed, so data of both two feet were combined for analysis. Suggested by Hopkins, typical error and re-test reliability needed to be calculated, where the typical error needed to be normalized in order to compare within groups. Then the coefficient of variance $(\mathrm{CoV})$ was calculated by dividing the typical error by mean value of the group. Test-retest reliability was evaluated by Intraclass correlation coefficient (ICC). The ICC's evaluation standard is that if ICC $<0.5$, reliability is poor; if $0.5<\mathrm{ICC}<0.75$, reliability is medium; if ICC $>0.75$, reliability is good. Reliabilities were calculated for three groups individually: three trials, four trials and five trials. All the statistical models were operated under the SPSS software with significant level of 0.05 and confidence interval of $95 \%$.

\section{RESULTS}

In terms of CP, with the increase of test times, ICC of PP increased while those of PTIrel and CArel was lowered; in terms of TD, ICC of each pressure variable successively improved. A similar tendency was found for CoV (Figure 2).

In terms of entire feet of $\mathrm{CP}$, outcomes of five measurements showed that ICC of CArel was the highest (ICC $=0.990, \mathrm{CoV}=9.4 \%, p=0.000)$, then was the PP (ICC $=0.760, \mathrm{CoV}=48.9 \%, p=0.000)$ and the PTIrel (ICC $=0.714, \mathrm{CoV}=44.5 \%, p=0.000)$; whereas, $\mathrm{CP}$ also showed that with exception of ICC of CArel under each region was found to be higher than $0.8(p=$

\section{Prelucrarea datelor}

S-a aplicat modelul cu trei zone şi regiunile plantare au fost redefinite de software-ul sistemului: antepiciorul (inclusiv cele cinci degete şi cinci metatarsiene), zona mediană a piciorului şi retropiciorul. Apoi s-au calculat parametri precum presiunea maximă (PP) $\left(\mathrm{N} / \mathrm{cm}^{2}\right)$, integrala timp-presiune (PTI) $\left(\mathrm{S}^{*} \mathrm{~N} / \mathrm{cm}^{2}\right)$ şi zona de contact (CA) $\left(\mathrm{cm}^{2}\right)$ în fiecare zonă. Pentru a face comparaţii în cadrul grupului, s-a efectuat o prelucrare de normalizare pentru CA şi PTI: CA relativă $($ CArel $)=100 \times(C A(X i) / \Sigma C A$ $(\mathrm{Xi})$ ); PTI relativă $(\mathrm{PTIrel})=100 \times(\mathrm{PTI}(\mathrm{Xi}) / \Sigma \mathrm{PTI}(\mathrm{Xi}))$, unde $\mathrm{Xi}$ indică una dintre regiunile plantare.

\section{Analiza statistică}

Mai întâi, reprezentarea grafică $Q-Q$ a arătat că toate datele testate au urmat distribuţia normală; rezultatele testului $\mathrm{t}$ independent au confirmat că nu au existat diferenţe semnificative, astfel încât s-au combinat pentru analiză datele ambelor picioare. Potrivit lui Hopkins, a fost necesară calcularea erorii tipice şi a fiabilităţii retestării, unde eroarea tipică trebuia normalizată pentru a face comparaţii în cadrul grupurilor. Apoi s-a calculat coeficientul de variaţie (CoV) prin împărţirea erorii tipice la valoarea medie a grupului. Fiabilitatea testare-retestare a fost evaluată prin coeficientul de corelaţie intra-clasă (ICC). Standardul de evaluare a ICC indică faptul că la ICC $<0,5$, fiabilitatea este slabă; la $0,5<$ ICC $<0,75$, fiabilitatea este medie; la ICC $>0.75$, fiabilitatea este bună. Fiabilitatea a fost calculată individual pentru trei grupe: trei teste, patru teste şi cinci teste. Toate modelele statistice au fost prelucrate cu software-ul SPSS cu nivel de semnificaţie de 0,05 şi interval de încredere de $95 \%$.

\section{REZULTATE}

La copiii cu PC, odată cu mărirea numărului de teste, ICC al PP a crescut în timp ce ICC al PTIrel şi CArel a scăzut; la copii cu TD, ICC al fiecărei variabile de presiune s-a îmbunătăţit succesiv. O tendinţă similară sa constatat pentru CoV (Figura 2).

În ceea ce priveşte analiza piciorului întreg la copiii cu PC, rezultatele a cinci măsurători au arătat că ICC al CArel a avut valoarea cea mai mare (ICC $=0,990$, $\mathrm{CoV}=9,4 \%, p=0,000)$, apoi au urmat PP $(I C C=0,760$, $\mathrm{CoV}=48,9 \%, \mathrm{p}=0,000)$ şi PTIrel $($ ICC $=0,714, \mathrm{CoV}=$ $44,5 \%, p=0,000)$; de asemenea, la copiii cu PC, cu excepţia faptului că ICC al CArel în fiecare regiune s-a 
0.000), those of PP and PTIrel were proved to be forefoot $<$ hindfoot < midfoot; meanwhile CoV of those two variables were higher than $50 \%$, particularly under hindfoot area. Similar outcomes were observed at TD group, where reliability sequences were CArel (ICC = 0.994, CoV $=9.4 \%, p=0.000)>$ PTIrel $(I C C=0.900$, CoV $=$ $23.3 \%, p=0.000)>P P(I C C=0.880, \mathrm{CoV}=23.9 \%, p=$ 0.000 ); besides, three pressure variables under each area were demonstrated to be good to excellent as well, and $\mathrm{CoV}$ values were also in a reasonable range (CoV<30\%) (Table 2).

Overall, reliability of $\mathrm{CP}$ was recorded to be moderate (ICC $>0.5$, CoV $<50 \%, p=0.000$ for all parameters); further, those of TD were good to excellent (ICC $>0.8, \mathrm{CoV}<25 \%, \mathrm{p}=0.000$ for all parameters). dovedit a fi mai mare decât $0,8(p=0,000)$, secvenţa valorilor ICC la PP şi PTIrel a fost antepicior < retropicior $<$ partea mediană; CoV al acestor două variabile a fost peste $50 \%$, mai ales în zona retropiciorului. Rezultate similare au fost observate la grupa de copii TD, unde secvenţele de fiabilitate au fost CArel (ICC $=0,994, \mathrm{CoV}$ $=9,4 \%, p=0,000)>$ PTIrel $($ ICC $=0,900, \mathrm{CoV}=23,3 \%, p=$ $0,000)>P P(I C C=0,880, \operatorname{CoV}=23,9 \%, p=0,000) ;$ în plus, cele trei variabile de presiune în fiecare zonă au fost bune spre excelente, iar CoV a fost, de asemenea, într-un interval rezonabil (CoV< $30 \%$ ) (Tabelul 2).

În general, fiabilitatea PC a fost moderată (ICC > $0,5, \mathrm{CoV}<50 \%, p=0,000$ pentru toţi parametrii); mai mult, valorile grupei TD au fost bune spre excelente (ICC $>0,8, \mathrm{CoV}<25 \%, p=0,000$ pentru toţi parametrii).
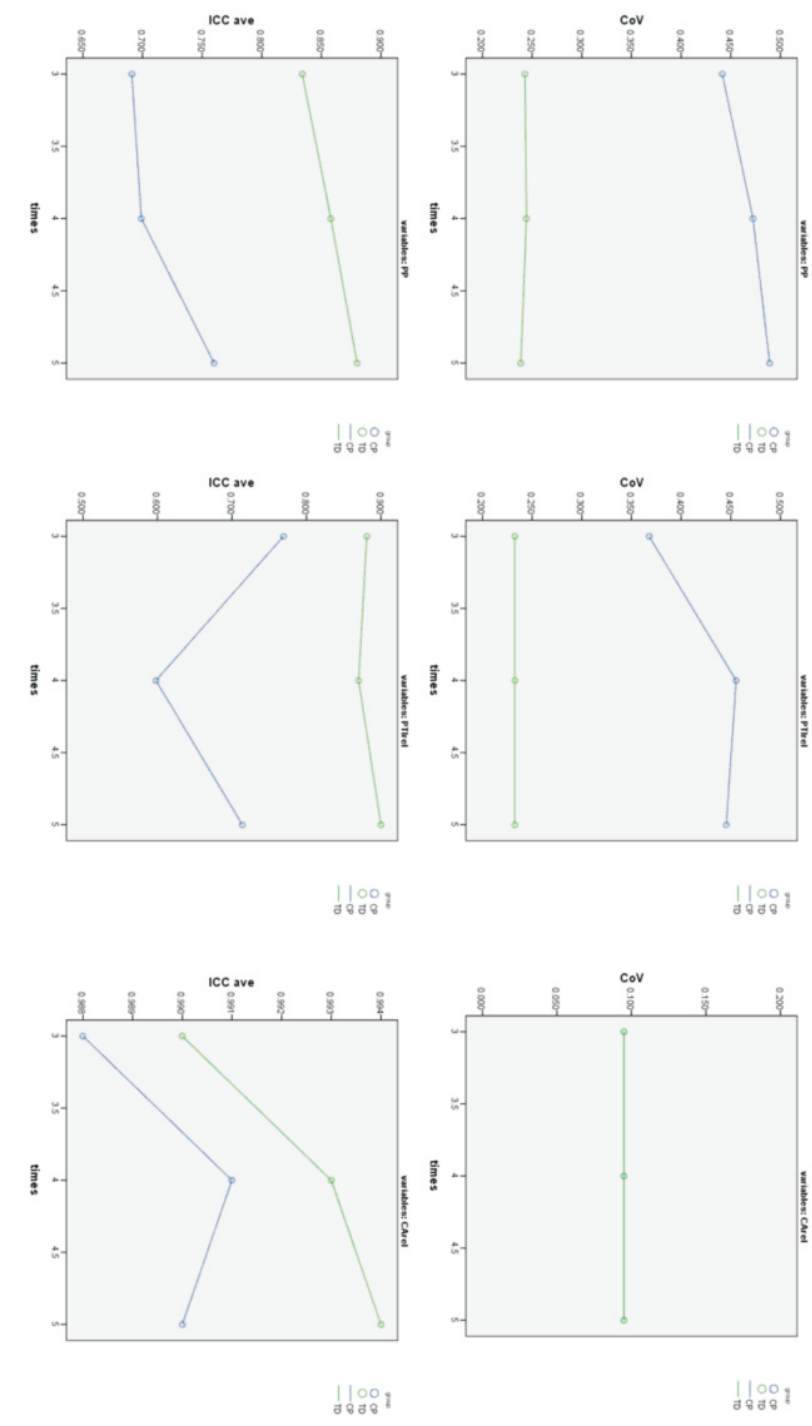

Figure 2. Changes of CoV and ICC in three, four, five measurements

Figura 2. Modificările CoV şi ICC în urma măsurătorilor efectuate de trei, patru şi cinci ori 
Table 2: Reliability of pressure variables under each region of CP and TD based on five times test Tabelul 2: Fiabilitatea variabilelor de presiune în fiecare regiune din grupele PC şi TD pe baza celor cinci măsurători

\begin{tabular}{|c|c|c|c|c|c|c|c|c|c|c|}
\hline $\begin{array}{l}\text { Variables } \\
\text { Variabile }\end{array}$ & $\begin{array}{l}\text { Group } \\
\text { Grupă }\end{array}$ & $\begin{array}{l}\text { Regions } \\
\text { Regiuni }\end{array}$ & $\begin{array}{l}\text { Mean } \\
\text { Medie }\end{array}$ & $\begin{array}{l}\text { Residual mean } \\
\text { square } \\
\text { Media pătrată a } \\
\text { reziduurilor }\end{array}$ & $\mathrm{CoV}$ & $\begin{array}{l}\text { ICC } \\
\text { ave } \\
\text { ICC } \\
\text { mediu }\end{array}$ & $\begin{array}{l}\text { Lower } \\
\text { bound } \\
\text { Limită } \\
\text { inferioară }\end{array}$ & $\begin{array}{l}\text { Upper } \\
\text { bound } \\
\text { Limită } \\
\text { superioară }\end{array}$ & $\mathrm{F}$ test & sig \\
\hline PP & $\mathrm{CP}$ & $\begin{array}{l}\text { Forefoot } \\
\text { Antepicior }\end{array}$ & 3.204 & 1.267 & $35.1 \%$ & 0.389 & -0.168 & 0.728 & 1.638 & 0.068 \\
\hline PP & $\mathrm{CP}$ & $\begin{array}{l}\text { Midfoot } \\
\text { Parte } \\
\text { mediană }\end{array}$ & 3.12 & 1.467 & $38.8 \%$ & 0.671 & 0.371 & 0.853 & 3.041 & $0.000 * *$ \\
\hline PP & $\mathrm{CP}$ & $\begin{array}{l}\text { Hindfoot } \\
\text { Retropicior }\end{array}$ & 4.637 & 7.063 & $57.3 \%$ & 0.74 & 0.504 & 0.0884 & 3.853 & $0.000 * *$ \\
\hline PTIrel & $\mathrm{CP}$ & $\begin{array}{l}\text { Forefoot } \\
\text { Antepicior }\end{array}$ & 0.399 & 0.026 & $40.4 \%$ & 0.526 & 0.092 & 0.788 & 2.108 & $0.012^{*}$ \\
\hline PTIrel & $\mathrm{CP}$ & $\begin{array}{l}\text { Midfoot } \\
\text { Parte } \\
\text { mediană }\end{array}$ & 0.318 & 0.013 & $35.9 \%$ & 0.849 & 0.711 & 0.933 & 6.614 & $0.000 * *$ \\
\hline PTIrel & $\mathrm{CP}$ & $\begin{array}{l}\text { Hindfoot } \\
\text { Retropicior }\end{array}$ & 0.283 & 0.027 & $58.1 \%$ & 0.593 & 0.222 & 0.819 & 2.458 & $0.003^{*}$ \\
\hline CArel & $\mathrm{CP}$ & $\begin{array}{l}\text { Forefoot } \\
\text { Antepicior }\end{array}$ & 0.538 & 0.001 & $5.9 \%$ & 0.854 & 0.721 & 0.935 & 6.867 & $0.000 * *$ \\
\hline CArel & $\mathrm{CP}$ & $\begin{array}{l}\text { Midfoot } \\
\text { Parte } \\
\text { mediană }\end{array}$ & 0.312 & 0.002 & $14.3 \%$ & 0.86 & 0.731 & 0.937 & 7.12 & $0.000 * *$ \\
\hline CArel & $\mathrm{CP}$ & $\begin{array}{l}\text { Hindfoot } \\
\text { Retropicior }\end{array}$ & 0.15 & 0.001 & $21.1 \%$ & 0.868 & 0.747 & 0.941 & 7.551 & $0.000 * *$ \\
\hline PP & TD & $\begin{array}{l}\text { Forefoot } \\
\text { Antepicior }\end{array}$ & 4.038 & 0.383 & $15.3 \%$ & 0.805 & 0.628 & 0.913 & 5.14 & $0.000 * *$ \\
\hline PP & TD & $\begin{array}{l}\text { Midfoot } \\
\text { Parte } \\
\text { mediană }\end{array}$ & 4.287 & 0.483 & $16.2 \%$ & 0.874 & 0.759 & 0.944 & 7.94 & $0.000 * *$ \\
\hline PP & TD & $\begin{array}{l}\text { Hindfoot } \\
\text { Retropicior }\end{array}$ & 5.659 & 2.825 & $29.7 \%$ & 0.845 & 0.703 & 0.931 & 6.435 & $0.000 * *$ \\
\hline PTIrel & TD & $\begin{array}{l}\text { Forefoot } \\
\text { Antepicior }\end{array}$ & 0.405 & 0.007 & $20.7 \%$ & 0.833 & 0.681 & 0.926 & 5.999 & $0.000 * *$ \\
\hline PTIrel & TD & $\begin{array}{l}\text { Midfoot } \\
\text { Parte } \\
\text { mediană }\end{array}$ & 0.285 & 0.004 & $22.2 \%$ & 0.867 & 0.746 & 0.941 & 7.52 & $0.000 * *$ \\
\hline PTIrel & TD & $\begin{array}{l}\text { Hindfoot } \\
\text { Retropicior }\end{array}$ & 0.31 & 0.007 & $27.0 \%$ & 0.905 & 0.818 & 0.958 & 10.498 & $0.000 * *$ \\
\hline CArel & TD & $\begin{array}{l}\text { Forefoot } \\
\text { Antepicior }\end{array}$ & 0.575 & 0.000 & $0.0 \%$ & 0.924 & 0.855 & 0.966 & 13.214 & $0.000 * *$ \\
\hline CArel & TD & $\begin{array}{l}\text { Midfoot } \\
\text { Parte } \\
\text { mediană }\end{array}$ & 0.282 & 0.001 & $11.2 \%$ & 0.862 & 0.736 & 0.938 & 7.242 & $0.000 * *$ \\
\hline CArel & TD & $\begin{array}{l}\text { Hindfoot } \\
\text { Retropicior }\end{array}$ & 0.143 & 0.001 & $22.1 \%$ & 0.784 & 0.586 & 0.904 & 4.624 & $0.000 * *$ \\
\hline
\end{tabular}

*significant differences were lower than 0.05

*diferenţele semnificative au fost mai mici decât 0,05

**significant differences were lower than 0.001

**diferenţele semnificative au fost mai mici decât 0,001 


\section{DISCCUSION}

In our study, $18 \mathrm{CP}$ and 36 TD counterparts were recruited and their plantar pressure distribution was measured by Footscan plate system; then the reliability of pressure variables under each region was evaluated. Our outcomes show that at first, in terms of $\mathrm{CP}$, with the increasing of test times, ICC of PP increased while those of PTIrel and CArel were lowered; in terms of TD, ICC of each pressure variable was successively improved; afterwards, in aspect of entire feet of CP and based on five times measurement, reliability of CArel was the best in the three areas; whereas PP and PTIrel were demonstrated to be less reliable and they were proved to be relatively repeatable at MF area. At last, reliability of TD group was shown to be good for all pressure variables under all three regions.

In terms of CP, Chen et al. [14] investigated the reliability of time-spatial parameters of $45 \mathrm{CP}$ children (26 hemiplegia and 19 diplegia); their results demonstrated that ICC of each parameter were higher than 0.9, when five trials for hemiplegia and four trials for diplegia were measured. Anne Brit Sorsdahl [6] analyzed reliability of the electronic walkway in the application of gait pattern of CP children; they also showed that $70 \%$ parameters were recorded with good reliability. Another similar study concerning subjects with foot deformity, Jacques Riad et al. [16] summarized that although a good reliable outcome could be obtained for the subjects without motion limit, those with limited motion or CP children required more trials in order to obtain a more reliable outcome.

Regarding other pressure systems, Gurney [8] reported a study which measured plantar pressure of nine subjects over five successive days and their CoV and ICC showed that in the higher loading areas, such as forefoot and hindfoot area, pressure outcomes were reliable and repeatable; whereas, under the toe and mid foot, where lower loading was obtained, reliability and repeatability were inferior. This finding was confirmed with Zammit et al. [3]; additionally, Zammit et al. implied that as the hallux functioned as

\section{DISCUTII}

În studiul nostru, s-au recrutat 18 subiecţi cu PC şi 36 subiecţi dezvoltaţi normal şi s-a măsurat distribuţia presiunii plantare utilizând sistemul Footscan; apoi s-a evaluat fiabilitatea variabilelor de presiune în fiecare regiune. Rezultatele noastre arată că la început, la subiecţii cu PC, la creşterea numărului de măsurători, ICC al PP a crescut, în timp ce ICC al PTIrel şi CArel a scăzut; la subiecţii dezvoltaţi normal, ICC pentru fiecare variabilă de presiune s-a îmbunătăţit succesiv; după aceea, în ceea ce priveşte analiza piciorului întreg la subiecţii cu PC în urma celor cinci măsurători, fiabilitatea CArel a fost cea mai bună în cele trei regiuni; PP şi PTIrel au fost mai puţin fiabile şi s-au dovedit a fi relativ repetabile în zona MF. În cele din urmă, fiabilitatea grupei TD s-a dovedit a fi bună pentru toate variabilele de presiune în toate cele trei regiuni.

În ceea ce priveşte subiecţii cu PC, Chen şi colab. [14] au investigat fiabilitatea parametrilor temporalspaţiali la 45 de copii cu PC ( 26 cu hemiplegie şi $19 \mathrm{cu}$ diplegie), iar rezultatele lor au demonstrat că valoarea ICC obţinută pentru fiecare parametru a fost mai mare decât 0,9 , atunci când s-au efectuat cinci măsurători pentru hemiplegie şi patru măsurători pentru diplegie. Anne Brit Sorsdahl [6] a analizat fiabilitatea plăcii electronice la analiza tiparului de mers la copiii cu PC, raportând că $70 \%$ din parametri s-au înregistrat cu fiabilitate bună. Un alt studiu similar privind subiecţii cu deformări ale piciorului, Jacques Riad şi colab. [16] au relatat că, deşi s-ar putea obţine un rezultat de încredere pentru subiecţi fără limitări de mişcare, cei cu limitări de mişcare sau copiii cu PC necesită mai multe măsurători pentru a obţine rezultate mai fiabile.

În ceea ce priveşte alte sisteme de măsurare a presiunii, Gurney [8] a raportat un studiu în care a măsurat presiunea plantară a nouă subiecţi timp de cinci zile consecutive, iar valorile $\mathrm{CoV}$ şi ICC au arătat că, în zona cu încărcare mai mare, cum ar fi antepiciorul şi retropiciorul, rezultatele presiunii au fost fiabile şi repetabile; la degete şi în zona mediană s-a obţinut o încărcare mai mică, iar fiabilitatea şi repetabilitatea au fost inferioare. Această constatare a fost confirmată de Zammit şi colab. [3]; în plus, aceştia au sugerat că 
pushing off, this area was received as a moderate CoV and ICC; Nevertheless, opposite outcomes were also reported in the study of Ramanathan, A.K. [1], whose finding showed that results of hallux area were unstable.

In our study, according to the outcomes of comparison within three, four, and five measurements, with the test trials increasing and exception of PTIrel, reliability of PP and CArel of CP increased. We suggested that as both the contact time and loading force were unstable, PTIrel was unreliable. Thereby our outcomes confirmed our first hypothesis, where reliability of two variables was found to increase upon increasing the test times. Further, our finding was also consistent with that of Chen et al.

Since gait pattern of $\mathrm{CP}$ varied for each subject, such as footflat and forefoot contact, some plantar areas did not participate in the gait cycle, three-mask model strategies were adopted and reliability of those areas were considered in our study. Our results indicated that the best reliable data were obtained for CArel in all three areas; while the reliability of PP and PTIrel under heel and forefoot were poor, this might be caused by the unstable gait patterns. Hence, our second and third hypotheses were partially confirmed.

At last, although ICC and CoV of CP varied with various regions, $44.4 \%$ ICC items were larger than $0.75,33.3 \%$ of those were higher than 0.5 , and $77.8 \%$ CoV items were lower than 50\%, thereby, we suggested that Footscan pressure plate was reliable in the study of CP subjects in terms of plantar pressure distribution.

Although positive outcomes were obtained, limitations still existed and need to be comprehended with caution: 1) subjects were asked to walk with their own selected speed and the factor of speed was potentially correlated with PP and PTIrel; 2) a small sample strategy was adopted in this study and this might contribute to a larger vibration range of data. halucele ar funcţiona ca mecanism de împingere, această regiune a avut valori moderate pentru CoV şi ICC. Cu toate acestea, rezultate opuse au fost raportate în studiul lui Ramanathan, A.K. [1], care a constatat că rezultatele zonei halucelui au fost instabile.

În studiul nostru, în conformitate cu rezultatele comparaţiei celor trei, patru şi cinci măsurători, la creşterea numărului de măsurători, cu excepţia PTIrel, fiabilitatea PP şi CArel la subiecţii cu PC a crescut. Noi am sugerat că, întrucât atât timpul de contact, cât şi forţa de încărcare au fost instabile, valorile PTIrel nu au fost fiabile. Astfel rezultatele noastre au confirmat prima ipoteză, în care fiabilitatea a două variabile a crescut cu mărirea numărului de măsurători. Mai mult, constatarea noastră a fost, de asemenea, în concordanţă cu aceea a lui Chen şi colab.

Întrucât tiparul de mers al subiecţilor cu PC a variat de la unul la altul, cum ar fi faza de sprijin şi contactul antepiciorului, şi unele zone plantare nu au participat la ciclul de mers, s-a adoptat modelul cu trei zone şi s-a studiat fiabilitatea acestor zone. Rezultatele noastre au indicat că cele mai bune date fiabile au fost obţinute pentru CArel în toate cele trei regiuni; în timp ce fiabilitatea PP şi PTIrel la călcâi şi antepicior a fost slabă, acest lucru putând fi cauzat de tiparul instabil al mersului. Prin urmare, a doua şi a treia noastră ipoteză au fost parţial confirmate.

În cele din urmă, deşi ICC şi CoV la subiecţii cu PC au variat în diverse regiuni, 44,4\% din elementele ICC au fost mai mari decât 0,75, 33,3\% dintre acestea au fost mai mari decât 0,5 , şi $77,8 \%$ din elementele CoV au fost mai mici decât 50\%; astfel, am sugerat că placa de presiune Footscan a fost de încredere în studiul subiecţilor cu PC în ceea ce priveşte distribuţia presiunii plantare.

Deşi s-au obţinut rezultate pozitive, au existat şi limitări şi acestea trebuie să fie luate în considerare cu precauţie: 1) subiecţii au fost rugaţi să meargă cu o viteză la alegere, iar factorul de viteză a fost corelat potenţial cu PP şi PTIrel; 2) în acest studiu s-a adoptat o strategie pentru un eşantion mic şi acest lucru ar putea contribui la mai mare variabilitate a datelor. 


\section{CONCLUSION}

Overall, reliability of Footscan plate system in the study of CP patients was moderate in most variables and under most plantar regions. Thereby, by increasing the test times to no less than five, this protocol could be applied in the clinical and scientific investigation of CP children.

\section{Acknowledgement}

The authors appreciate all the children and their parents participating in this study; meanwhile we also acknowledge the financial support from the funding of national postdoctoral project (150620) and "Fund for Young Researchers" (2014SCU11029) of Sichuan University.

\section{CONCLUZII}

Per ansamblu, fiabilitatea sistemului Footscan la studierea pacienţilor cu PC a fost moderată în cazul majorităţii variabilelor şi în majoritatea regiunilor plantare. Astfel, prin creşterea numărului de testări la nu mai puţin de cinci, acest protocol ar putea fi aplicat în investigaţia clinică şi ştiinţifică a copiilor cu PC.

Mulţumiri

Autorii doresc să mulţumească tuturor copiilor şi părinţilor care au participat la acest studiu; în acelaşi timp ne exprimăm recunoştinţa pentru sprijinul financiar din cadrul proiectului naţional postdoctoral (150620) şi programului „Fonduri pentru cercetători tineri” (2014SCU11029) din cadrul Universităţii Sichuan.

\section{REFERENCES}

1. Ramanathan, A.K., Kiran, P., Arnold, G.P., Wang, W., Abboud, R.J., Foot Ankle Surg, 2010, 16, 2, 70-73.

2. Maetzler, M., Bochdansky, T., Abboud, R.J., Gait Posture, 2010, 32, 3, 391-394.

3. Zammit, G.V., Menz, H.B., Munteanu, S.E., J Foot Ankle Res, 2010, 3, 11.

4. Jiong, Y., Nian, L., Qingsong, Z., Chinese Journal of Practical Pediatrics, 2007, 22, 231-233.

5. Xiaofeng, J., Xueyan, H., Chin J Rehabil Theory Pract, 2009, 15, 65-66.

6. Sorsdahl, A.B., Gait Posture, 2008, 27, 43-50.

7. Hai, H., Anyan, Z., Dongfeng, H. et al., Chinese Journal of Rehabilitation Medicine, 2007, 22, 44-47.

8. Gurney, J.K., Kersting, U.G. et al., Gait Posture, 2008, 27, 706-709.

9. Low, D.C., Gait Posture, 2010, 32, 664-666.

10. De Cock, A., Vanrenterghem, J., et al., Gait Posture, 2008, 27, 669-675.

11. Zhou, J., Song, Y., Xu, B., Chen, W., Revista de Pielarie Incaltaminte (Leather and Footwear Journal), 2014, 14, 3, 135-146.

12. Zhou, J., Zhang, Y., Chen, W., Xu, B., Revista de Pielarie Incaltaminte (Leather and Footwear Journal), 2014, 14, 4, 205-214.

13. Zhou, J., Li, T., Xu, B., Chen, W., Revista de Pielarie Incaltaminte (Leather and Footwear Journal), 2015, 15, 1, 3-14.

14. Longwei, C., Shuwei, L., Jue, W., Lei, S., Chin J Sports Med, 2012, 31, 303-307.

15. Bus, S.A., Clinical Biomechanics, 2005, 20, 892-899.

16. Jacques Riad, S.C., Henley, S., Miller, F., Gait Posture, 2006, 24S, S98-S289.

17. Hopkins, W.G., Sports Medicine, 2000, 30, 1-15.

Article received/Data primirii articolului: 08.07.2015

Accepted/Acceptat la data: 19.11.2015 